\title{
Immune responses to gp82 provide protection against mucosal Trypanosoma cruzi infection
}

\author{
Christopher S Eickhoff' ${ }^{1}$, Olivia K Giddings ${ }^{1}$, Nobuko Yoshida ${ }^{3}$, Daniel F Hoft ${ }^{1,2}{ }^{+}$
}

${ }^{1}$ Department of Internal Medicine 2Department of Molecular Microbiology, Saint Louis University, 1100 S. Grand Blvd, Saint Louis, MO, 63104 USA ${ }^{3}$ Departamento de Microbiologia, Imunologia e Parasitologia, Escola Paulista de Medicina, Universidade Federal de São Paulo, São Paulo, SP, Brasil

The potential use of the Trypanosoma cruzi metacyclic trypomastigote (MT) stage-specific molecule glycoprotein-82 (gp82) as a vaccine target has not been fully explored. We show that the opsonization of T. cruzi MT with gp82-specific antibody prior to mucosal challenge significantly reduces parasite infectivity. In addition, we investigated the immune responses as well as the systemic and mucosal protective immunity induced by intranasal CpGadjuvanted gp82 vaccination. Spleen cells from mice immunized with CpG-gp82 proliferated and secreted IFN- $\gamma$ in a dose-dependent manner in response to in vitro stimulation with gp 82 and parasite lysate. More importantly, these CpG-gp82-immunized mice were significantly protected from a biologically relevant oral parasite challenge.

Key words: Trypanosoma cruzi - mucosal immunity - vaccines

Trypanosoma cruzi, the causative agent of Chagas disease, infects more than 11 million people and kills an estimated 50,000 individuals annually (CDC 2007). Up to $30 \%$ of infected individuals will develop complications from Chagas disease, which include cardiomyopathy and the mega-syndromes (megaesophagus and megacolon). In humans, natural infection occurs primarily through contact with $T$. cruzi-contaminated reduviid excreta, usually through breaks in the skin or mucosal surfaces. Recent reports of large-scale outbreaks following food or drink contamination demonstrate the dangers of oral T. cruzi infection (Dias et al. 2008, Beltrão et al. 2009, Nóbrega et al. 2009, Pereira et al. 2009, Alarcón de Noya et al. 2010).

Because parasite proteins are expressed differentially in distinct $T$. cruzi life stages, it may be difficult to develop a vaccine that establishes sterile immunity. Glycoprotein-82 (gp82), a stage-specific protein present on the surface of $T$. cruzi metacyclic trypomastigotes (MT), has been shown to bind to gastric mucin and to facilitate the $\mathrm{Ca}^{2+}$-signaling activity necessary for parasite internalization (Yoshida 2006, 2009). Isolates of $T$. cruzi that lack gp82 expression are much less efficient at infecting mice orally than those that express high levels of gp82 (Cortez et al. 2003). Antibodies that bind to gp82 inhibit the in vitro infection of epithelial cells (Neira et al. 2003). Although gp 82 is only expressed by MT, it could be a good target for immune responses aimed at providing protection against initial immune invasion and intracellular replication cycles.

Financial support: NIH (RO1 AI040196) (to DFH), FAPESP (to NY) + Corresponding author: hoftdf@slu.edu

Received 6 January 2010

Accepted 13 May 2010
It is well documented that type 1 immune responses are critical for protection against both mucosal and systemic T. cruzi infection (Hoft et al. 2000, Hoft \& Eickhoff 2005, Rodrigues et al. 2009). The CpG motifs present within ssDNA are known to induce type $1 \mathrm{im}$ mune responses mediated by toll-like receptor 9 (TLR9) stimulation and have been used as adjuvants safely and effectively in mice and humans (Krieg 2000, Dumais et al. 2002, Cooper et al. 2005). Several independent groups have shown the induction of protective immune responses against lethal T. cruzi challenge using $\mathrm{CpG}$ mixed with whole $T$. cruzi lysate or various recombinant $T$. cruzi proteins (Frank et al. 2003, Araújo et al. 2005, Hoft et al. 2007). In this study, we tested a mucosal T. cruzi vaccine containing the gp82 protein. We show that intranasal vaccination with $\mathrm{CpG}+\mathrm{gp} 82$ induces protective immune responses against mucosal $T$. cruzi challenge.

\section{MATERIALS AND METHODS}

Parasites and mice - Female Harlan Sprague Dawley $\mathrm{BALB} / \mathrm{c}$ mice aged 6-8 weeks were used throughout these studies and housed in Association for Assessment and Accreditation of Laboratory Animal Care International (AAALAC) accredited facilities. Tulahuén strain T. cruzi insect-derived MT (IMT), culture-derived MT and blood form trypomastigotes (BFT) were prepared as previously described (Hoft \& Eickhoff 2005, Giddings et al. 2006).

Opsonization and T. cruzi challenges - IMT mixed 1:1 with $0.5 \mathrm{mg} / \mathrm{mL}$ MAb-3F6 (gp82-specific monoclonal antibody) or isotype control (Sigma, St. Louis, MO, USA) were incubated at RT for $30 \mathrm{~min}$ prior to conjunctival or oral challenges, which were performed as previously described (Hoft 1996, Giddings et al. 2006).

Immunization with CpG-gp82 - Recombinant gp82 and control glutathione S-transferase (GST) proteins were prepared as previously described (Santori et al. 1996). Oligodeoxynucleotide (ODN) \# 1826 (5'-TCCATGACGTTCCTGACGTT-3') containing CpG motifs (underlined) and control ODN were purchased from Coley Pharmaceuticals 
(Wellesley, MA). BALB/c mice were immunized intranasally twice, two weeks apart, using $10 \mu \mathrm{g} \mathrm{CpG} 1826$ mixed with 2-10 $\mu \mathrm{g}$ gp82 (or control GST).

Measurement of vaccine induced immunity - Four weeks after immunization, spleen cells from vaccinated mice were stimulated at $1 \times 10^{6} \mathrm{cells} / \mathrm{mL}$ in round-bottom 96-well plates with $0.4-2.0 \mu \mathrm{g} / \mathrm{mL}$ recombinant gp82 or control GST protein. Spleen cells $\left(4 \times 10^{6} / \mathrm{mL}\right)$ from these same immunized mice were stimulated in 24-well plates with medium or $10 \mu \mathrm{g} / \mathrm{mL} \mathrm{T}$. cruzi lysate (Hoft \& Eickhoff 2005). Three days later, proliferation and IFN- $\gamma$ secretion were assessed as previously described (Hoft \& Eickhoff 2005).

Determination of parasite infectivity - Four weeks after the final immunization, some mice ( $\mathrm{n}=5$ /group) were challenged with 5,000 BFT subcutaneously and survival was monitored as a means of analyzing systemic protective immunity. Other groups of mice $(n=5-8 /$ group) were challenged orally with 1,000 IMT and parasite replication in mucosal tissues was determined using T. cruzi-specific real-time polymerase chain reaction (PCR). Mice were harvested 11-13 days after mucosal $T$. cruzi challenge and the levels of parasite replication were measured in draining lymph node and tissue DNA samples using $T$. cruzi-specific real-time PCR as previously described (Giddings et al. 2006, Hoft et al. 2007). Briefly, DNA was purified from splenic and gastric lymph node tissue (after oral MT challenge) and from submandibular lymph nodes (after conjunctival MT challenge) using a commercially available kit (DNeasy, QIAGEN). T. cruzi-specific real-time PCR was performed using $100 \mathrm{ng}$ of purified DNA per reaction.

Ethics - All animal studies were performed in AAALAC accredited facilities (NIH Assurance A3225$01)$. In addition, all mouse studies were approved by the Institutional Animal Care and Use Committee/Animal Care Committee at Saint Louis University.

\section{RESULTS}

Gp82-specific antibodies reduce T. cruzi infectivity - Our group has shown that the opsonization of $T$. cruzi MT with faecal extracts containing T. cruzi-specific secretory IgA can reduce parasite infectivity after conjunctival challenge (Giddings et al. 2006). In the present study, we incubated Tulahuén strain IMT with a control or gp82-specific MAb-3F6. The opsonized parasites were then placed on the conjunctiva of anesthetized mice or fed orally to naïve mice. Two weeks later, mice were sacrificed and parasite replication was determined by real-time PCR in tissues taken from sites of early infection. In mice challenged conjunctivally with antigp82-opsonized parasites, significantly reduced parasite DNA was detectible in the lymph nodes that drain the site of initial invasion in the nasal cavity relative to mice infected with control opsonized parasites $(\mathrm{p}<0.04$ by Mann-Whitney U test) (Fig. 1A).

Similarly, mice challenged orally with anti-gp82-opsonized parasites had $\sim 4$-fold lower amounts of $T$. cruzi DNA recoverable at the site of initial invasion in gastric tissue compared with controls (Fig. 1B). These results
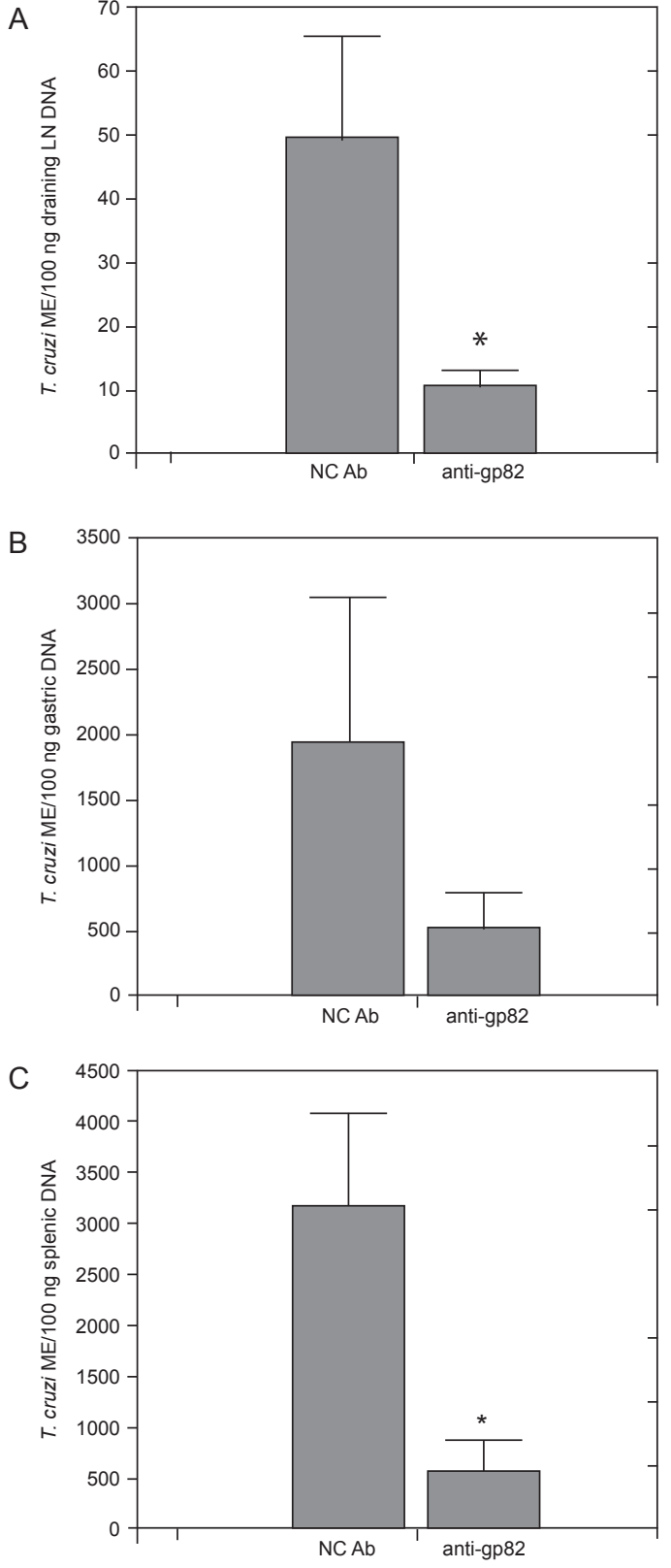

Fig. 1: decreased parasite infectivity after opsonization with glycoprotein-82 (gp82)-specific antibodies. Insect-derived metacyclic trypomastigotes (IMT) were incubated with control or anti-gp82 specific monoclonal antibodies and then either placed on the conjunctiva of anesthetized mice (A) or fed orally to BALB/c mice (B, C). DNA extracted 11-13 days later from local draining tissues (parotid and submandibular lymph nodes (LN) from conjunctivally challenged mice or gastric DNA from orally challenged animals) or distant sites (spleen) were used in Trypanosoma cruzi specific real-time polymerase chain reaction assays. In panel A, the numbers of $T$. cruzi molecular equivalents (ME) per $100 \mathrm{ng}$ of DNA is significantly lower (asterisk means $\mathrm{p}<0.04$ by Mann-Whitney $U$ test) in anti-gp 82 opsonized MT-infected mice than in control opsonized MT-infected mice. Similarly, mice challenged orally had markedly reduced amounts of $T$. cruzi DNA present in gastric samples after opsonization with anti-gp82 monoclonal antibody (MAb)-3F6 as compared to controls (B). In addition, systemic spread of parasites was inhibited by opsonization with anti-gp82, as seen in panel $\mathrm{C}$ where reduced parasite DNA was detectable in spleens recovered from mice that were infected with anti-gp82 MAb 3F6opsonized MT compared with control opsonized MT (asterisk means $\mathrm{p}<0.02$ Mann-Whitney U Test). NC: negative control. 
show that gp82-specific antibodies can reduce infectivity at the initial sites of parasite mucosal invasion after both conjunctival and oral $T$. cruzi challenges. In addition, significantly decreased amounts of parasite DNA were detected in splenic DNA samples isolated from mice challenged orally with anti-gp82 opsonized MT compared with mice challenged with control opsonized MT ( $p<0.02$ by Mann-Whitney U test) (Fig. 1C). Thus, the systemic spread of parasite infection can be curtailed by opsonization with gp82-specific antibody, suggesting that disseminated disease from $T$. cruzi can be limited by the neutralization of initial parasite infectivity.

Intranasal vaccination with CpG-gp82 induces T. cruzi-specific type 1 immune responses - Systemic immunization with alum + gp 82 has previously been shown to induce immune responses that were capable of reducing parasitaemia after systemic MT challenge (Santori et al. 1996). In the present study, we intranazally vaccinated mice with gp82 adjuvanted with CpG, a TLR-9 agonist. Four weeks following the second and final immunization, spleen cells from immunized animals were harvested and stimulated with antigen. The spleen cells from mice immunized with CpG-gp82 proliferated in a dose-dependant manner in response to recombinant gp82 antigen stimulation in vitro (Fig. 2A). Spleen cells from these same vaccinated animals produced IFN- $\gamma$ upon in vitro stimulation with gp82 (Fig. 2B). Additionally, spleen cells from mice immunized with CpG-gp82 produced IFN- $\gamma$ after in vitro stimulation with whole T. cruzi lysate (Fig. 2C). The production of IFN- $\gamma$ by spleen cells from gp82-immunized mice after stimulation with $T$. cruzi antigens is especially important because type $1 \mathrm{immune}$ responses have been shown to be critical in protecting against both systemic and mucosal $T$. cruzi challenges (Hoft \& Eickhoff 2002, 2005).

Intranasal CpG-gp82 isimmunization fails to protect mice against systemic BFT challenge - In order to assess protective immunity induced by CpG-gp 82 immunization, immunized mice were challenged systemically or mucosally four weeks after their second and final vaccination. For systemic survival studies, 5,000 BFT diluted in $100 \mu \mathrm{L}$ PBS were injected subcutaneously at the base of the tail. As seen in Fig. 3A, mice immunized with CpGgp82 failed to survive lethal T. cruzi challenge and time to death was not prolonged.

CpG-gp82 immunization results in protection after a biologically relevant mucosal MT challenge - In order to study mucosal protection, immunized mice were challenged orally with 1,000 IMT. DNA was extracted from stomachs 11-13 days after oral challenge and infection levels were measured using T. cruzi-specific real-time PCR. The gastric DNA obtained from CpG-gp82-vaccinated mice contained significantly reduced amounts of T. cruzi DNA compared with gastric DNA samples from $\mathrm{CpG}$-negative control GST-immunized mice ( $\mathrm{p}<0.05$ by Mann-Whitney U test) (Fig. 3B).

\section{DISCUSSION}

All four T. cruzi life stages have been studied for gp 82 expression by simple IFA, Western blot and, more recently, by proteome analysis (Teixeira \& Yoshida 1986, Atwood
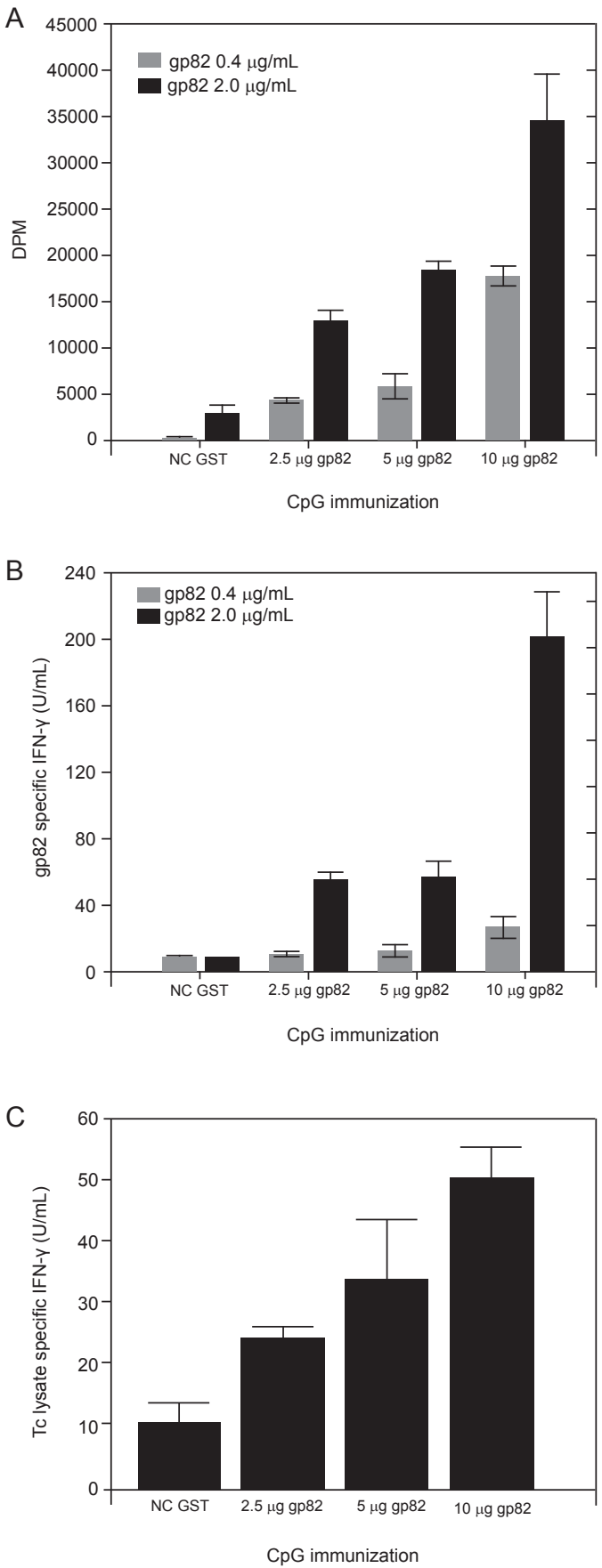

Fig. 2: type 1 mediated immune responses induced by intranasal $\mathrm{CpG}-$ glycoprotein-82 (gp82) immunization. BALB/c mice were immunized twice two weeks apart with $10 \mu \mathrm{g} \mathrm{CpG} 1826$ mixed with $2.5-10 \mu \mathrm{g}$ gp82 or control glutathione-S-transferase (GST) protein. Four weeks later, spleen cells were removed and stimulated in vitro for three days with control protein, recombinant gp82, or Trypanosoma cruzi lysate, after which time proliferative responses were detected using ${ }^{3} \mathrm{H}$-thymidine incorporation and IFN- $\gamma$ secretion measured via ELISA. Negative control stimulation values were subtracted. Spleen cells from mice immunized with $\mathrm{CpG}-\mathrm{gp} 82$ proliferated and produced IFN- $\gamma$ in a dose dependent manner in response to recombinant gp 82 stimulation in vitro (A, B). As seen in panel C, mice immunized with CpG-gp82 also produced IFN- $\gamma$ in response to T. cruzi lysate stimulation. Based on these immune response results, $10 \mu \mathrm{g}$ gp 82 was chosen for immunization and challenge experiments to assess vaccine-induced protective immunity DPM: disintegrations per minute; NC: negative control. 
A

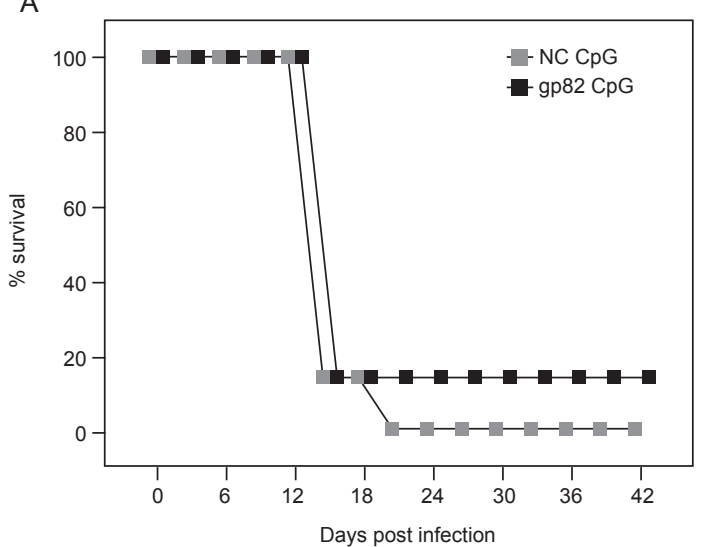

B

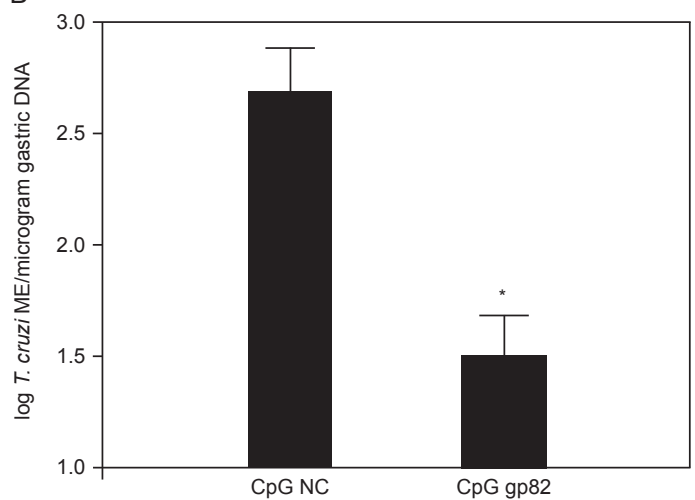

Fig. 3: mucosal protective immunity induced by intranasal CpG-glycoprotein-82 (gp82) immunization. BALB/c mice were immunized intranasally twice two weeks apart with $10 \mu \mathrm{g}$ CpG 1826 mixed with $10 \mu \mathrm{g}$ gp82 or control glutathione-S-transferase protein. Four weeks after the final immunization, groups of mice were challenged with 5,000 blood form trypomastigotes (BFT) subcutaneously (A) or with 1,000 insectderived metacyclic trypomastigotes orally (B). Control and CpG-gp82 immunized mice failed to survive challenge with a normally lethal dose of Trypanosoma cruzi BFT (A). However, as compared with the control immunized group, mice vaccinated with $\mathrm{CpG}$-gp 82 had significantly lower amounts of T. cruzi DNA detectable in gastric DNA samples taken 12 days post infection as determined by T. cruzi specific real-time polymerase chain reaction (B) (asterisk means $\mathrm{p}<0.01$ using MannWhitney U test). ME: molecular equivalente; NC: negative control.

et al. 2005, CDC 2007, The T. cruzi proteome database 2007). It is clear that gp 82 is only expressed by T. cruzi MT and not by T. cruzi intracellular amastigotes (AMA) or BFT. The critical role of gp82 in parasite mucosal epithelial invasion makes this $T$. cruzi glycoprotein an ideal target for mucosal immune responses. It is a highly conserved molecule present in nearly all $T$. cruzi isolates, including strains Y, CL, F, Tulahuén, G and others (Yoshida 2006). Epimastigotes do not express gp82 and are non-infectious (Manque et al. 2003). In addition, gp82-deficient MT are defective in mucosal infectivity in mice after oral delivery (Cortez et al. 2003). The binding of gp82 to gastric mucin induces intracellular $\mathrm{Ca}^{+}$increases dependent on ATP consumption, an increase which appears to be required for parasite internalization. The interaction and binding of gp82 to gastric mucin may explain earlier findings in which MT, but not BFT, efficiently infected mice after oral challenge (Hoft 1996). Previous work has shown that antibodies directed against gp 82 significantly reduce parasite infectivity both in vitro and after oral in vivo challenges. We have extended these findings by (i) showing that antigp82 Abs can block T. cruzi infection via both conjunctival and oral routes and (ii) demonstrating that a mucosal vaccine can significantly reduce mucosal infection after oral $T$. cruzi challenge. It seems likely that the gp82 gastric mucin-binding epitope overlaps with the gp82-specific MAb-3F6 epitope. Alternatively, immune responses directed against gp82 could block the induction of $\mathrm{Ca}^{+} / \mathrm{ATP}-$ mediated membrane repair responses, thereby preventing active parasite internalization.

It has been reported that mice challenged systemically with MT after gp82 vaccination develop lower parasitaemia compared with control immunized mice (Santori et al. 1996). However, those data were generated using an MT challenge as opposed to a BFT challenge and the endpoint analysis was peak parasitaemia rather than death. In our study, we found no evidence of protection against systemic BFT challenge. Stage-specific gp82 expression on MT (not AMA or BFT) is the most logical explanation for these apparently conflicting data because immune responses directed towards MT may not be effective when BFT not expressing gp82 are used for parasite challenge. Because Santori et al. (1996) used an MT challenge, the gp82-specific immunity that was induced could have partially protected against this initial infecting stage. However, we challenged systemically with BFT that did not express gp 82 and, therefore, the gp82-specific immunity is likely to have been irrelevant in recognizing this infection.

Many studies investigating T. cruzi vaccine development have focused on the induction of systemic immune responses and have utilized lethal, but perhaps biologically irrelevant, challenge models. As new natural infections in humans occur through contact with IMTcontaminated reduviid excreta, we must investigate the effects of vaccine-induced immunity on these types of infections. Our results showing gp82 vaccine-induced mucosal protection after a biologically relevant IMT challenge are one such example of these types of studies. Future T. cruzi vaccines should encode antigens that can induce both mucosal and systemic immunity. The gp82 antigen could facilitate the induction of optimal mucosal protection, but other antigens will be required for the induction of optimal systemic protection.

The prevalence of human T. cruzi infection increases dramatically with the presence of infected dogs in households (Cohen \& Gürtler 2001). The removal of infected dog reservoirs alone can reduce the threat of human T. cruzi transmission. Previous studies on dogs immunized with live-attenuated $T$. cruzi epimastigotes provided partial protection against natural T. cruzi infection (Basombrio et al. 1993). Currently, it may be more feasible to develop a vaccine aimed at preventing systemic and mucosal infection of domesticated animals rather than humans. Our data clearly show that immunization with gp82 induces significant mucosal protection and could be useful in vaccines designed to prevent mucosal infection in dogs. 
Other vaccines composed of $T$. cruzi proteins expressed by BFT and/or AMA (including TS and ASP-2) induce strong T-cell responses and systemic protection in mice (Fujimura et al. 2001, Araújo et al. 2005). A vaccine composed of several stage-specific proteins could perhaps lead to optimal protective immunity by inducing immune responses that are relevant for both mucosal and systemic protection. Focusing future vaccine strategies on domestic animals would be much safer than vaccinating humans and could interrupt transmission in households. Thus, a multi-component vaccine that includes gp 82 and is aimed at preventing T. cruzi infection in dogs should be explored.

\section{ACKNOWLEDGEMENTS}

To Vanessa D Atayde, for preparation and purification of recombinant gp82.

\section{REFERENCES}

Alarcón de Noya B, Díaz-Bello Z, Colmenares C, Ruiz-Guevara R, Mauriello L, Zavala-Jaspe R, Suarez JA, Abate T, Naranjo L, Paiva M, Rivas L, Castro J, Márques J, Mendoza I, Acquatella H, Torres J, Noya O 2010. Large urban outbreak of orally acquired acute Chagas disease at a school in Caracas, Venezuela. J Infect Dis 201: 1308-1315.

Araújo AF, de Alencar BC, Vasconcelos JR, Hiyane MI, Marinho CR, Penido ML, Boscardin SB, Hoft DF, Gazzinelli RT, Rodrigues MM 2005. CD8 $8^{+}$-T-cell-dependent control of Trypanosoma cruzi infection in a highly susceptible mouse strain after immunization with recombinant proteins based on amastigote surface protein 2 . Infect Immun 73: 6017-6025.

Atwood JA 3rd, Weatherly DB, Minning TA, Bundy B, Cavola C, Opperdoes FR, Orlando R, Tarleton RL 2005. The Trypanosoma cruzi proteome. Science 309: 473-476.

Basombrio MA, Segura MA, Mora MC, Gomez L 1993. Field trial of vaccination against American trypanosomiasis (Chagas' disease) in dogs. Am J Trop Med Hyg 49: 143-151.

Beltrão H de B, Cerroni M de P, Freitas DR, Pinto AY, Valente V da C, Valente SA, Costa E de G, Sobel J 2009. Investigation of two outbreaks of suspected oral transmission of acute Chagas disease in the Amazon region, Para state, Brazil, in 2007. Trop Doct 39: 231-232.

CDC - Centers for Disease Control and Prevention 2007. Blood donor screening for Chagas disease - United States, 2006-2007. MMWR Morb Mortal Wkly Rep 56: 141-143.

Cohen JE, Gürtler RE 2001. Modeling household transmission of American trypanosomiasis. Science 293: 694-698.

Cooper CL, Davis HL, Angel JB, Morris ML, Elfer SM, Seguin I, Krieg AM, Cameron DW 2005. CPG 7909 adjuvant improves hepatitis B virus vaccine seroprotection in antiretroviral-treated HIV-infected adults. AIDS 19: 1473-1479.

Cortez M, Neira I, Ferreira D, Luquetti AO, Rassi A, Atayde VD, Yoshida N 2003. Infection by Trypanosoma cruzi metacyclic forms deficient in gp82 but expressing a related surface molecule, gp30. Infect Immun 71: 6184-6191.

Dias JP, Bastos C, Araújo E, Mascarenhas AV, Martins Netto E, Grassi F, Silva M, Tatto E, Mendonça J, Araújo RF, Shikanai-Yasuda MA, Aras R 2008. Acute Chagas disease outbreak associated with oral transmission. Rev Soc Bras Med Trop 41: 296-300.

Dumais N, Patrick A, Moss RB, Davis HL, Rosenthal KL 2002. Mucosal immunization with inactivated human immunodeficiency virus plus $\mathrm{CpG}$ oligodeoxynucleotides induces genital immune responses and protection against intravaginal challenge. J Infect Dis 186: 1098-1105.

Frank FM, Petray PB, Cazorla SI, Muñoz MC, Corral RS, Malchiodi EL 2003. Use of a purified Trypanosoma cruzi antigen and CpG oligodeoxynucleotides for immunoprotection against a lethal challenge with trypomastigotes. Vaccine 22: 77-86.

Fujimura AE, Kinoshita SS, Pereira-Chioccola VL, Rodrigues MM 2001. DNA sequences encoding $\mathrm{CD} 4^{+}$and $\mathrm{CD} 8^{+} \mathrm{T}$-cell epitopes are important for efficient protective immunity induced by DNA vaccination with a Trypanosoma cruzi gene. Infect Immun 69: 5477-5486.

Giddings OK, Eickhoff CS, Smith TJ, Bryant LA, Hoft DF 2006. Anatomical route of invasion and protective mucosal immunity in Trypanosoma cruzi conjunctival infection. Infect Immun 74: 5549-5560.

Hoft DF 1996. Differential mucosal infectivity of different life stages of Trypanosoma cruzi. Am J Trop Med Hyg 55: 360-364.

Hoft DF, Eickhoff CS 2002. Type 1 immunity provides optimal protection against both mucosal and systemic Trypanosoma cruzi challenges. Infect Immun 70: 6715-6725.

Hoft DF, Eickhoff CS 2005. Type 1 immunity provides both optimal mucosal and systemic protection against a mucosally invasive, intracellular pathogen. Infect Immun 73: 4934-4940.

Hoft DF, Eickhoff CS, Giddings OK, Vasconcelos JR, Rodrigues MM 2007. Trans-sialidase recombinant protein mixed with CpG motifcontaining oligodeoxynucleotide induces protective mucosal and systemic Trypanosoma cruzi immunity involving CD8 ${ }^{+} \mathrm{CTL}$ and B cell-mediated cross-priming. J Immunol 179: 6889-6900.

Hoft DF, Schnapp AR, Eickhoff CS, Roodman ST 2000. Involvement of $\mathrm{CD}^{+}$Th1 cells in systemic immunity protective against primary and secondary challenges with Trypanosoma cruzi. Infect Immun 68: 197-204.

Krieg AM 2000. Immune effects and mechanisms of action of $\mathrm{CpG}$ motifs. Vaccine 19: 618-622.

Manque PM, Neira I, Atayde VD, Cordero E, Ferreira AT, da Silveira JF, Ramirez M, Yoshida N 2003. Cell adhesion and $\mathrm{Ca}_{2}{ }^{+}$signaling activity in stably transfected Trypanosoma cruzi epimastigotes expressing the metacyclic stage-specific surface molecule gp82. Infect Immun 71: 1561-1565.

Neira I, Silva FA, Cortez M, Yoshida N 2003. Involvement of Trypanosoma cruzi metacyclic trypomastigote surface molecule gp 82 in adhesion to gastric mucin and invasion of epithelial cells. Infect Immun 71: 557-561.

Nóbrega AA, Garcia MH, Tatto E, Obara MT, Costa E, Sobel J, Araujo WN 2009. Oral transmission of Chagas disease by consumption of açai palm fruit, Brazil. Emerg Infect Dis 15: 653-655.

Pereira KS, Schmidt FL, Guaraldo AM, Franco RM, Dias VL, Passos LA 2009. Chagas' disease as a foodborne illness. J Food Prot 72: 441-446.

Rodrigues MM, de Alencar BC, Claser C, Tzelepis F, Silveira EL, Haolla FA, Dominguez MR, Vasconcelos JR 2009. Swimming against the current: genetic vaccination against Trypanosoma cruzi infection in mice. Mem Inst Oswaldo Cruz 104 (Suppl. I): 281-287.

Santori FR, Paranhos-Bacalla GS, Franco DA Silveira J, Yamauchi LM, Araya JE, Yoshida N 1996. A recombinant protein based on the Trypanosoma cruzi metacyclic trypomastigote 82-kilodalton antigen that induces and effective immune response to acute infection. Infect Immun 64: 1093-1099.

Teixeira MM, Yoshida N 1986. Stage-specific surface antigens of metacyclic trypomastigotes of Trypanosoma cruzi identified by monoclonal antibodies. Mol Biochem Parasitol 18: 271-282.

The T. cruzi proteome database [database on the internet]. The T. cruzi proteome. Athens (GA): c 2007 - [cited January 2010]. Available from: kiwi.rcr.uga.edu/tcprot/.

Yoshida N 2006. Molecular basis of mammalian cell invasion by Trypanosoma cruzi. An Acad Bras Cienc 78: 87-111.

Yoshida N 2009. Molecular mechanisms of Trypanosoma cruzi infection by oral route. Mem Inst Oswaldo Cruz 104 (Suppl. I): 101-107. 